\title{
GJAI is a Prognostic Biomarker and Correlated with Immune Infiltrates in Colorectal Cancer
}

This article was published in the following Dove Press journal:

Cancer Management and Research

\author{
Wenjun $\mathrm{Hu}^{1,2, *}$ \\ Shuchun $\mathrm{Li}^{1,2, *}$ \\ Sen Zhang ${ }^{1,2}$ \\ Bowen Xie' \\ Minhua Zheng ${ }^{1,2}$ \\ Jing Sun ${ }^{1,2}$ \\ Xiao Yang ${ }^{1,2}$ \\ Lu Zang ${ }^{1,2}$
}

'Department of General Surgery, Ruijin Hospital, Shanghai Jiao Tong University School of Medicine, Shanghai, People's Republic of China; ${ }^{2}$ Shanghai Minimally Invasive Surgery Center, Ruijin Hospital, Shanghai Jiao Tong University School of Medicine, Shanghai, People's Republic of China

*These authors contributed equally to this work
Correspondence: Lu Zang; Xiao Yang Email zanglu@I63.com;

yangxiaol9890125@I63.com
Purpose: Previous studies found that gap junction alpha-1 protein (GJA1) was a potent tumor suppressor in colorectal cancer (CRC). We designed the present study to evaluate the clinical importance and molecular mechanisms of GJA1 in CRC.

Methods: Clinical and transcriptomic data from TCGA and GEO datasets were retrospectively collected. CRC patients were divided into two subgroups according to the expression level of GJA1 mRNA. Difference between survival time and response to neoadjuvant chemotherapy was then evaluated. Functional assays including wound-healing assay, transwell invasion assay and flow cytometry assay were performed to investigate the effects of GJA1 on invasive ability and response to chemotherapy drugs of CRC cells. Moreover, we explored the mechanisms of GJA1 by which it regulates CRC malignant phenotypes.

Results: The expression level of GJA1 was significantly higher in normal tissue than cancer tissue, indicating a tumor suppressive role of GJA1 in CRC. Patients with higher expression of GJA1 showed better prognosis than those with low GJA1 expression level. Consistently, overexpression of GJA1 suppressed the invasive ability of CRC cells while enhancing the sensitivity of CRC cells to oxaliplatin-induced apoptosis. Mechanically, we found that GJA1 suppressed the epithelial mesenchymal transition process. Moreover, GJA1 could modulating infiltrating levels of several immune cells in the tumor microenvironment.

Conclusion: These findings suggested that GJA1 was correlated with prognosis and immune infiltrating levels of CD8+ T cells, macrophages, neutrophils, and DCs in CRC. In addition, GJA1 expression contributes to regulation of tumor-associated macrophages (TAMs) and tumor infiltrating neutrophils (TINs) in CRC. These findings suggest that GJA1 is a promising biomarker for determining prognosis and immune infiltration in colorectal cancer.

Keywords: colorectal cancer, the gap junction channel protein, metastasis, chemotherapy, immune infiltrates

\section{Introduction}

Colorectal cancer (CRC) is the third most common cause of cancer-related deaths worldwide. ${ }^{1}$ The high relative mortality is largely due to the ability of CRC to metastasize to remote sites such as the liver and lung. ${ }^{2}$ Although the development of chemotherapy and surgical techniques has been reported, ${ }^{3}$ the prognosis of metastatic colorectal cancer still remains grim. Therefore, understanding the molecular mechanisms and identify novel treatment target are of great importance.

The initiation and progression of colorectal cancer is thought to result from the accumulation of genetic and epigenetic alterations such as oncogenes and tumor suppressor genes as well as epigenetic changes of additional genes. ${ }^{4}$ Gap junctions 
(GJs) are specific cell-to-cell channels and have an essential role in intercellular communication, adhesion, and cell proliferation by allowing direct exchange of small molecules $(<1 \mathrm{kD})$ and subsequently biologic signaling between adjacent cells. ${ }^{5,6}$ Among GJ family members, gap junction alpha-1 protein (GJA1, Connexin43) is the predominant one expressed in epithelial tissues. It has been shown that down-regulation of GJA1 can result in aggressive growth of breast and lung cancer. ${ }^{5-7}$ In contrast, forced expression of GJA1 in various tumor cells can restore normal cell phenotypes and well differentiation. ${ }^{8}$ These findings indicated a possible role of GJA1 as a tumor suppressor. However, there are limited studies focusing on the role of GJA1 in CRC. Therefore, understanding the mechanisms by which GJA1 regulates tumorigenesis or tumor progression may provide a potential target in cancer prevention and therapy.

Accumulating evidence has emphasized that the cross-talk between tumor cells and immune stromal cells is implicated in tumor progression, drug resistance, and clinical outcomes. ${ }^{9,10}$ A previous study defined various types of myeloid cells, such as tumor-associated macrophages (TAMs), tumor-infiltrated neutrophils (TINs), and T cells play important roles in tumor progression and implicated the clinical outcomes. ${ }^{11}$ For example, infiltration of cytotoxic $\mathrm{CD} 8+\mathrm{T}$ cells has been associated with a favorable prognosis of CRC patients, mainly because of the anti-tumor activity of CD8+ T cells. ${ }^{12}$ Moreover, gastric cancer patients with higher TINs were prone to overall survival benefit from postoperative adjuvant chemotherapy. ${ }^{13}$ However, the role of neutrophils in the tumor microenvironment and the clinical significance of neutrophil infiltration in peri-tumor tissues are controversial. A number of studies have shown that high levels of circulating neutrophils and neutrophil-to-lymphocyte ratio in peripheral blood are associated with poor prognosis in several types of cancer. ${ }^{14}$ The above results suggest that the roles and underlying mechanisms of tumor infiltrates in cancer progression and treatment need further investigation.

More importantly, it still remains unclear how genetic alterations in tumor cells could affect the infiltration of stromal cells. In this study, we found that GJA1 was downregulated in CRC and the loss of GJA1 is associated with a poorer prognosis and resistance to chemotherapy, confirming the clinical importance of GJA1 in CRC. Moreover, GJA1 could re-model the tumor micro-environment by affecting immune infiltrating cells, especially TINs, in the peri-tumor stromal tissue. Our work provided a novel marker for CRC treatment and shed new light on the mechanisms of CRC progression.

\section{Patients and Methods \\ Acquisition and Processing of TCGA Data}

Clinical and transcriptomic data of colon adenocarcinoma (COAD) and rectal carcinoma (READ) were collected from the GDC data portal (https://portal.gdc.cancer.gov/) of TCGA database. RNA expression level, copy number variation and methylation level of GJA1 were extracted and analyzed according to our study design.

\section{Patients and Specimens}

A cohort of 75 pairs of human CRC tissues and paired adjacent normal tissues were collected. The experimental study was approved by the Ethic Committee of Shanghai Ruijin Hospital (RJ20180126) and informed consent was obtained from all the cases enrolled in this study. All these patients were diagnosed as CRC pathologically and accepted radical surgery in the Minimally Invasive Surgery Centre, Ruijin Hospital, from 2013 to 2018 and were followed-up for 11 to 62 months. Patients who accepted preoperative treatment such as radiation or chemotherapy were excluded from our study. Cancer and paired normal tissues were embedded with paraffin and were tested with tissue microarray (TMA).

\section{Immunohistochemical (IHC) Analysis}

The IHC staining of TMA was performed according to the manufacturer's protocol (Immunostain SP kit, DakoCytomation, USA). Briefly, the tissue sections were deparaffinized and dehydrated, and then treated by $3 \%$ $\mathrm{H}_{2} \mathrm{O}_{2}$ at room temperature for 10 minutes to block endogenous peroxidase activity. Next, the tissue sections were incubated with citrate buffer for the retrieval of the antigen. Then the tissues were blocked with 3\% BSA at room temperature for 30 minutes, followed by incubating with proper antibody at $4^{\circ} \mathrm{C}$ overnight. Two independent pathologists blinded from any data of this study examined the IHC sections The scoring of GJA1 protein $(\mathrm{Cx} 43)$ was based on the proportion of staining $(0=0 \%, 1 \leq 25 \%, 2=$ $25 \%$ to $50 \%, 3=51 \%$ to $75 \%, 4 \geq 75 \%$ positive cells) and the staining intensity $(0=$ no staining, $1=$ weak, $2=$ moderate, 3 = strong). The scores for staining intensity and percentage were finally multiplied. An overall score of $\leq 6$ was defined as negative, while a score $>6$ was defined as positive. 


\section{Cell Culture and Transfection}

The human CRC cell lines used in our study were purchased from the American Type Culture Collection (ATCC, USA) and preserved in the lab. All these cells were cultured in RPMI-1640 medium with $10 \%$ fetal bovine serum (FBS), penicillin $\left(10^{7} \mathrm{U} / \mathrm{L}\right)$ and streptomycin $(10 \mathrm{mg} / \mathrm{L})$ and incubated at $37^{\circ} \mathrm{C}, 5 \% \mathrm{CO}_{2} . \mathrm{SW} 480$ and SW48 cells were transfected with a pEX-3 expression vector containing the gene encoding GJA1 (Genepharma, Shanghai, People's Reublic of China) or empty pEX-3 vector as control. Lipofectamine 2000 Transfection Reagent (Invitrogen, 11668019) was used according to the manufacturer's instructions.

\section{Western Blot}

Western blot analysis was performed as previously described. First, $100 \mu \mathrm{g}$ of protein was separated by $10 \%$ SDS-PAGE gel and transferred to PVDF membranes. The membranes were blocked with $5 \%$ bovine serum albumin (BSA) for 2 hours and then were incubated with primary antibodies at $4{ }^{\circ} \mathrm{C}$ overnight. The primary antibodies included Connexin 43 (C6219, Sigma Aldrich) and EMT sampler kit. Horseradish peroxidase-conjugated secondary antibodies were used and the protein bands were visualized by an enhanced chemiluminescence detection system (Amersham Bioscience, Piscataway, NJ, USA) according to the manufacturer's protocol.

\section{Wound-Healing and Cell Migration Assay}

For wound-healing assay, the cells were plated in 6-well plates and grown to approximately $90 \%$ confluence. The cells were subsequently starved of serum for 6 hours, and a wound was made by scratching the confluent monolayer with a $200 \mathrm{ul}$ pipette tip. The process of migration was observed and photographed 24 hours after the wounding. The wound size was measured randomly at three sites perpendicular to the wound. Each experiment was replicated three times. For cell migration assay, transwell chamber precoated with $1 \%$ collagen I was used $(8 \mu \mathrm{m}$ for a 24-well plate; Corning Costar, NY, USA) to perform cell migration assay. Then, 200 ul serum-free medium containing $10^{5} \mathrm{CRC}$ cells was added into the upper transwell chamber while $700 \mu \mathrm{L}$ RPMI-1640 medium with $20 \%$ fetal bovine serum (FBS) was added into the lower chamber. After $24 \mathrm{~h}$ incubation, migration cells were fixed with methanol and stained with $0.5 \%$ crystal violet for 30 minutes. Finally, the lower side of the chamber membrane were counted and photographed by microscope.

\section{Flow Cytometric Assay}

CRC cells were collected and washed with PBS buffer, and then centrifuged for 5 minutes at $1500 \mathrm{rpm}$. The cells were then resuspended in Binding Buffer and stained for 15 minutes at room temperature using the Annexin VFITC/PI Apoptosis Kit (BD, USA) according to the protocols. The number of apoptotic cells was detected using BD FACS Vantage System. PI-negative and annexin Vpositive cells were considered as early apoptotic (quadrant 3) cells, both PI- and annexin V-positive (quadrant 2) cells were late apoptotic cells. Three independent experiments were performed.

\section{Analysis of Immune Infiltrating Cells}

The TIMER online tool is a database which includes 10,897 samples across 32 cancer types from TCGA datasets to estimate the abundance of immune infiltrates. We analyzed GJA1 expression in colorectal cancer and the correlation of its expression with the tumor purity and abundance of immune infiltrates, including B cells, CD4 $+\mathrm{T}$ cells, CD8 $+\mathrm{T}$ cells, neutrophils, macrophages, and dendritic cells, via gene modules. Besides we also evaluated the correlations between GJA1 expression and gene markers of tumor-infiltrating immune cells to investigate the potential mechanisms by which GJA1 attracted these immune cells.

\section{Statistics}

Statistical analyses were performed using either a Student's $t$-test or a one-way analysis of variance (ANOVA), according to the characters of data. $\mathrm{R}$ software (3.5.2) and GraphPad Prism7 were used to perform data analysis and visualization.

\section{Results}

\section{Downregulation of GJAI Was Associated with Unfavorable Prognosis of CRC Patients}

To investigate the role of GJA1 in CRC, we first analyzed the expression of GJA1 in TCGA and GEO public dataset. As shown in Figure 1A and C, expression of GJA1 was lower in CRC tissue compared with normal tissues, which was consistent with published studies. Moreover, in M1 stage cases, GJA1 was drastically downregulated (Figure 1B). The above 
A

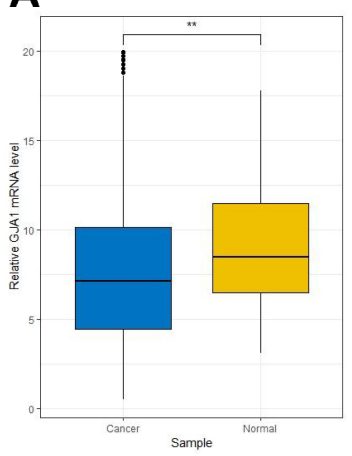

D

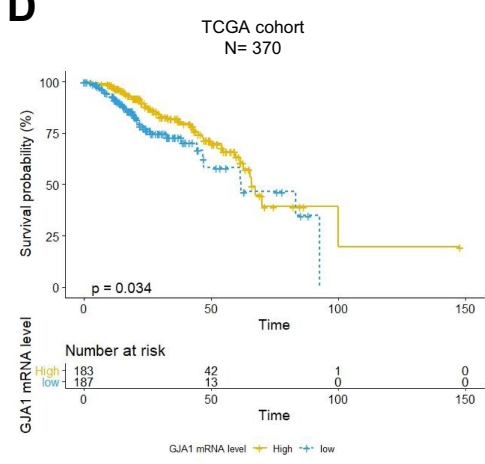

F

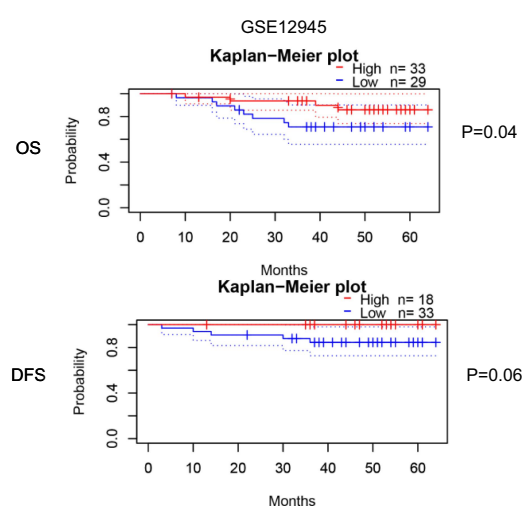

B

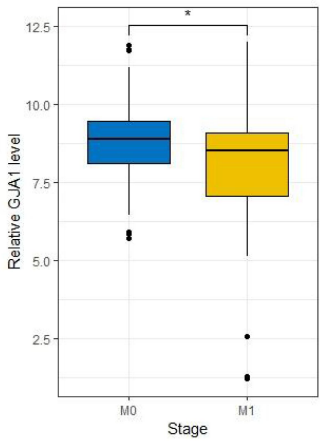

C

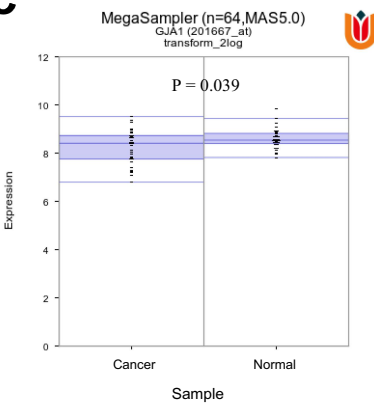

$\mathbf{E}$
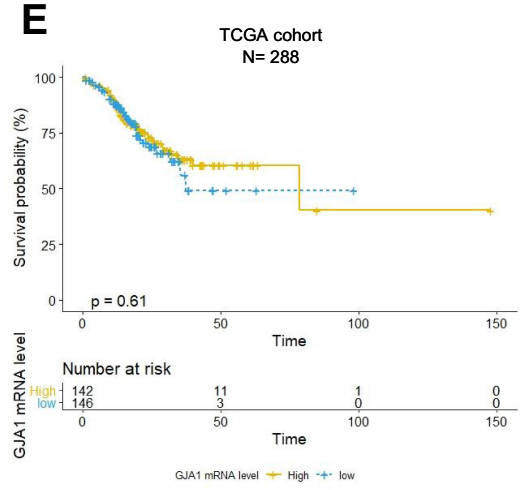

G

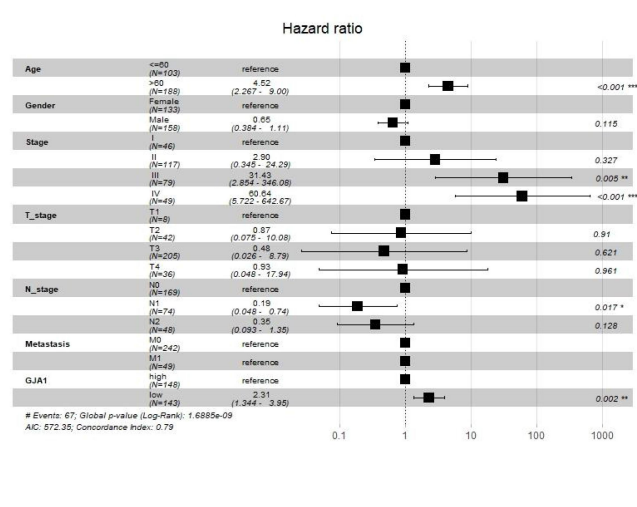

Figure I GJAI was a prognostic marker of CRC. (A and B), RNA-seq data showed that GJAI was downregulated in colorectal cancer tissues (A), especially in M stage cases (B). (C), Mega-sampler analysis of GEO dataset showed GJAI was significantly downregulated in CRC tissue. (D-F), Kaplan-Meier analysis of TCGA data (D and E) and GEO data (F) showed that high expression level of GJAI predicted better OS (D and upper panel of (F) and DFS (E and lower panel of (F). (G), multivariate Cox analysis showed that GJAI was an independent risk factor of OS. Data were obtained from at least three independent experiments and presented as mean \pm SD; $*<0.05$, ** $P<0.0$ I by Students' $t$-test or Mann-Whitney U-test.

results suggested that GJA1 was a potent tumor suppressor in $\mathrm{CRC}$ and was implicated in chemotherapy response. Next, we evaluated the prognostic value of GJA1. As expected, Kaplan-Meier analysis showed that CRC patients with high expression of GJA1 had significantly longer overall survival (OS) than those with lower GJA1 (Figure 1D). Also, the GJA1-high group tended to have a better disease-free survival (DFS) than the GJA1-low group (Figure 1E). Moreover, an external validation cohort from GEO dataset showed similar results that GJA1 expression was positively associated with better clinical outcomes of CRC patients (Figure 1F). In addition, multivariable Cox regression revealed GJA1 is an independent risk factor of CRC prognosis (Figure 1G).

Based on the results of bioinformatic analysis, we checked and analyzed GJA1 expression in CRC tissues from our cohort containing 75 patients to further investigate the clinical relevance of GJA1 in CRC. In accordance with the results of 
the bioinformatic analysis, expression of GJA1 was obviously lower in CRC tissue (Figure 2A-C). We then carried out correlation analysis to assess the relationship between GJA1 expression and clinical parameters by dividing patients into two groups: GJA1 negative $(\mathrm{N}=50)$ or GJA1 positive $(\mathrm{N}=25)$. Our data showed a negative correlation between GJA1 expression and $\mathrm{T}$ stage (Table 1). Because few stage-IV patients were enrolled in the cohort, we did not observe a significant correlation between GJA1 and metastasis. Also, Kaplan-Meier analysis revealed that patients in the GJA1 negative group had significantly poorer OS than those in the GJA1 positive group (log-rank $p=0.038$, Figure 2D).
A

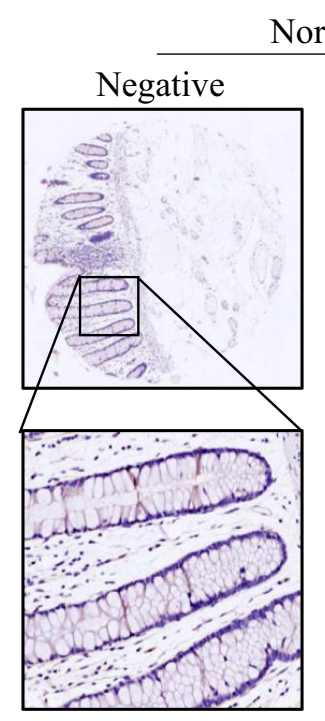

Normal

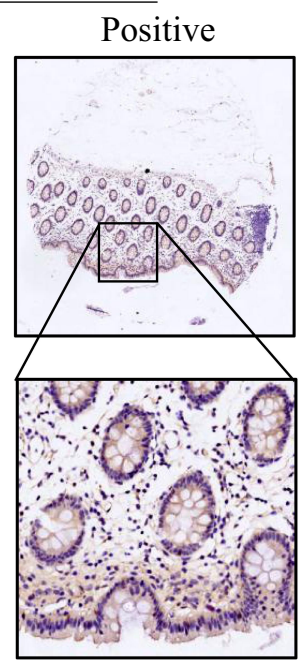

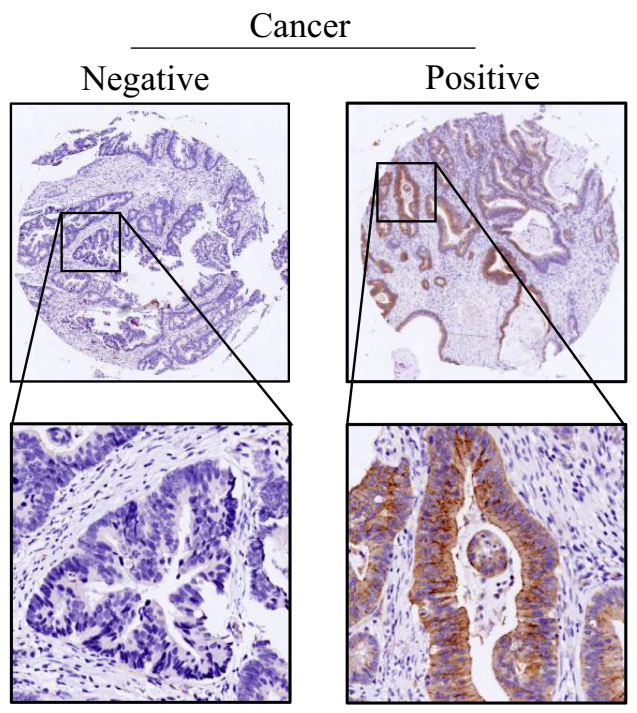

B

$$
\begin{array}{llllllllllllll}
\mathrm{N} 1 & \mathrm{~T} 1 & \mathrm{~N} 2 & \mathrm{~T} 2 & \mathrm{~N} 3 & \mathrm{~T} 3 & \mathrm{~N} 4 & \mathrm{~T} 4 & \mathrm{~N} 5 & \mathrm{~T} 5 & \mathrm{~N} 6 & \mathrm{~T} 6 & \mathrm{~N} 7 & \mathrm{~T} 7
\end{array}
$$

GJA1
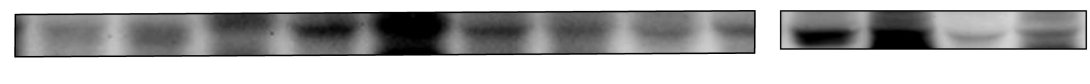

GAPDH
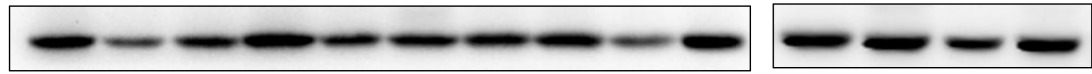

C

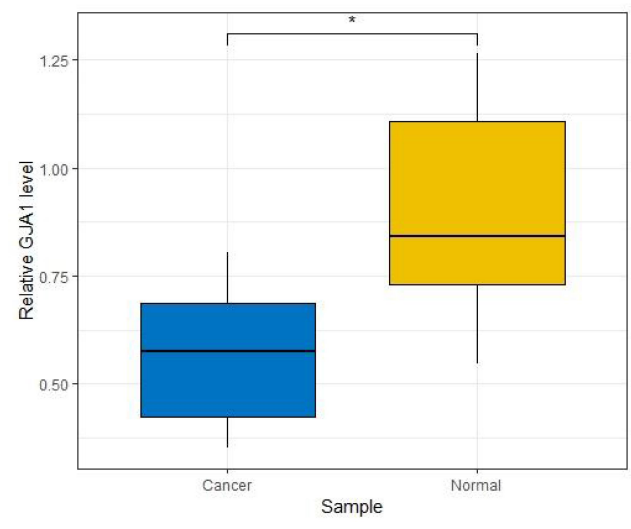

D

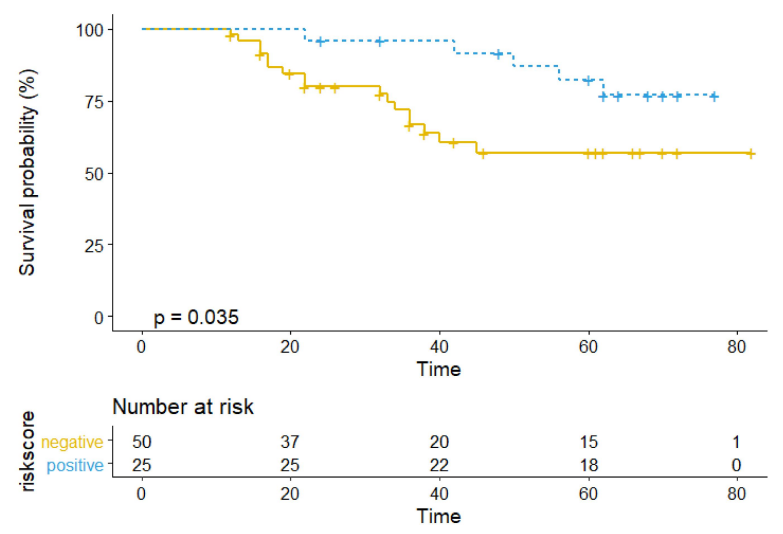

Figure 2 Expression level of GJAI in CRC patients from our cohorts. (A) Representative IHC staining of GJAI in CRC tissues and paired normal tissues form our cohort. (B) Expression level of GJAI in seven cases randomly selected from 75 CRC patients was evaluated by Western blot. (C) Statistical analysis of Western blot assay. (D) Kaplan-Meier analysis showed that CRC patients with positive GJAI expression had longer OS time. *P $<0.05$ by paired Students' $t$-test. 
Table I The Relation of GJAI and Clinicopathologic Parameters in 75 CRC Tissues

\begin{tabular}{|c|c|c|c|c|}
\hline \multirow[t]{2}{*}{ Parameters } & \multirow[t]{2}{*}{ Case } & \multicolumn{2}{|c|}{ GJAI Expression } & \multirow[t]{2}{*}{$P$-value } \\
\hline & & Positive & Negative & \\
\hline Age & & & & 0.8026 \\
\hline$<60$ & 30 & 9 & 21 & \\
\hline$\geq 60$ & 45 & 16 & 29 & \\
\hline Gender & & & & I \\
\hline Female & 46 & 15 & 31 & \\
\hline Male & 29 & 10 & 19 & \\
\hline T stage & & & & 0.039 \\
\hline $\mathrm{TI}+\mathrm{T} 2$ & 19 & 10 & 9 & \\
\hline $\mathrm{T} 3+\mathrm{T} 4$ & 56 & 15 & 41 & \\
\hline N stage & & & & 0.67 \\
\hline No & 43 & 13 & 30 & \\
\hline $\mathrm{N} 1+\mathrm{N} 2$ & 32 & 12 & 20 & \\
\hline M stage & & & & I \\
\hline Mo & 72 & 24 & 48 & \\
\hline MI & 3 & I & 2 & \\
\hline Location & & & & 0.588 \\
\hline Ascending colon & 22 & 9 & 13 & \\
\hline Descending colon & 9 & 2 & 7 & \\
\hline Sigmoid colon & 10 & 2 & 8 & \\
\hline Rectum & 35 & 13 & 22 & \\
\hline
\end{tabular}

Note: The stage of CRC was determined by AJCC guideline 8th edition.

Collectively, these data suggested that GJA1 might function as a prognostic marker for both OS and DFS of CRC patients.

\section{GJAI Suppressed Proliferation and Invasion While Promoted Apoptosis of CRC Cells}

To elucidate the underlying mechanisms by which GJA1 regulates the molecular phenotypes of CRC, we overexpressed GJA1 in two CRC cell lines: SW480 and HCT116 cells. As GJA1 is negatively correlated to T stage (Table 1) and $\mathrm{M}$ stage (Figure 1B), we presumed that GJA1 might suppress the progression, especially the invasion of CRC cells. To validate this hypothesis, the cell motility was firstly tested with wound healing assay. As shown, compared with the control group, GJA1-overexpressed cells migrated toward the wound more slowly than the negative control cells (Figure 3A, Figure S1A). This result was also confirmed by the transwell migration and invasion assay. We found that migrative and invasive abilities of CRC cells were significantly inhibited upon GJA1 ectopic expression (Figure 3B,
Figure S1B). Next, we detected the changes of epithelialmesenchymal transition (EMT) pathway, which was a symbol of cell invasion ability, after GJA1 overexpression using Western blot assay. The results showed that epithelial markers such as E-cadherin and ZO-1, were significantly upregulated in GJA1-overexpressed cells, while the expression of mesenchymal markers such as N-cadherin, Snail and Vimentin were decreased (Figure 3C). These results indicated that GJA1 could have impaired the migration and invasion of CRC cells through inhibiting the EMT process

In addition, to further illustrate the effects of GJA1 on cell viability, we extracted the expression profile of GSE10405 dataset and found GJA1 protein (Cx43) was downregulated in oxaliplatin-resistant cells (Figure 4A). Moreover, the expression level of GJA1 seemed to be lower in CRC patients who did not respond to oxaliplatin-based neoadjuvant therapy (Figure 4B, 4C), which suggested GJA1 might promote oxaliplatin-induced cell apoptosis. The established GJA1-overexpression cell models were incubated with oxaliplatin $(15 \mu \mathrm{m})$ for 12 hours and then analyzed with flow cytometric assay. As expected, the apoptotic rate was significantly higher in GJA1 cells than control cells (Figure 4D, Figure S1D). Taken together, these results demonstrate GJA1 acts as a potent tumor suppressor in CRC by suppressing invasion by promoting apoptosis of CRC cells.

\section{GJAI Modulated the Infiltration of Immune Cells of CRC Microenvironment}

Recent studies showed that tumor infiltrated immune cells configure the complicated microenvironment and influence tumor progression and treatment response. Gastric cancer patients with higher tumor infiltrating neutrophils (TINs) were prone to overall survival benefit from post-operative adjuvant chemotherapy. ${ }^{13}$ Other immune cells such as natural killer (NK) cell, dendrite cells (DC) and mast cells are also related to different phases of tumor immune response such as neoantigen presentation and finally affect the response of immunotherapy. ${ }^{15-17}$ We next asked whether GJA1 affected invasive ability and sensitivity of CRC cells to oxaliplatin by regulating the infiltrating level of tumor-associated immune cells. The correlations of GJA1 expression with immune infiltration levels in CRC were assessed by two independent sample cohorts generated by TIMER ${ }^{18}$ and TISIDB ${ }^{19}$ online tools. The results showed that GJA1 expression was positively correlated with infiltrating levels of $\mathrm{CD} 8+\mathrm{T}$ cells, macrophages, neutrophils, activated NK cells and DC (Figure 5A and B). 
A

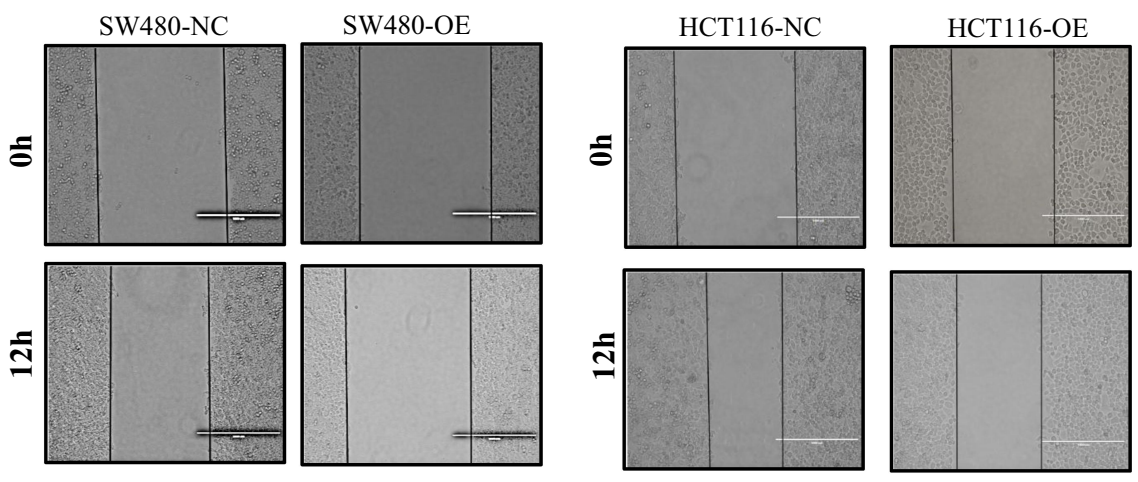

B

Migration Assay
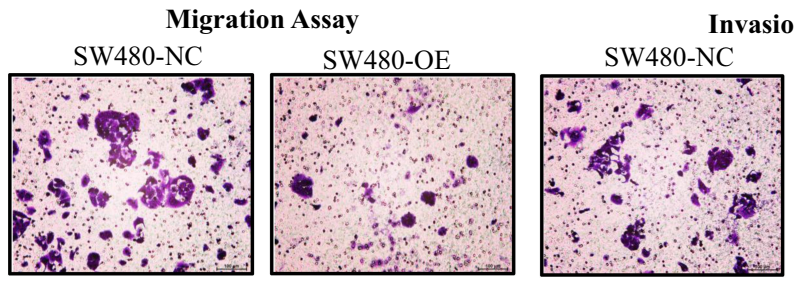

Invasion Assay

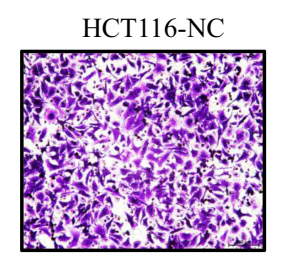

HCT116-OE

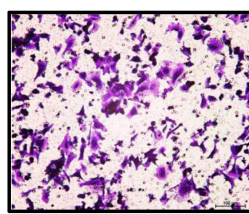

HCT116-NC
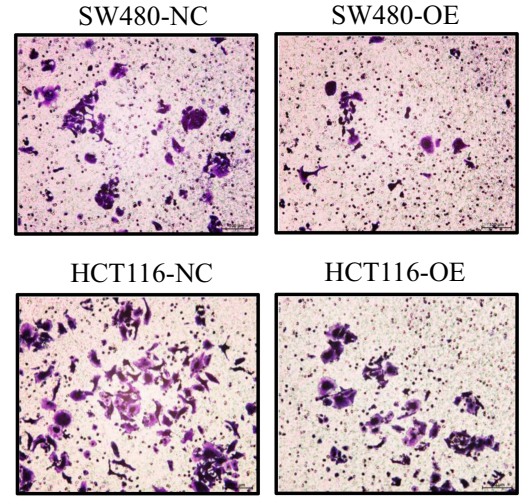

C

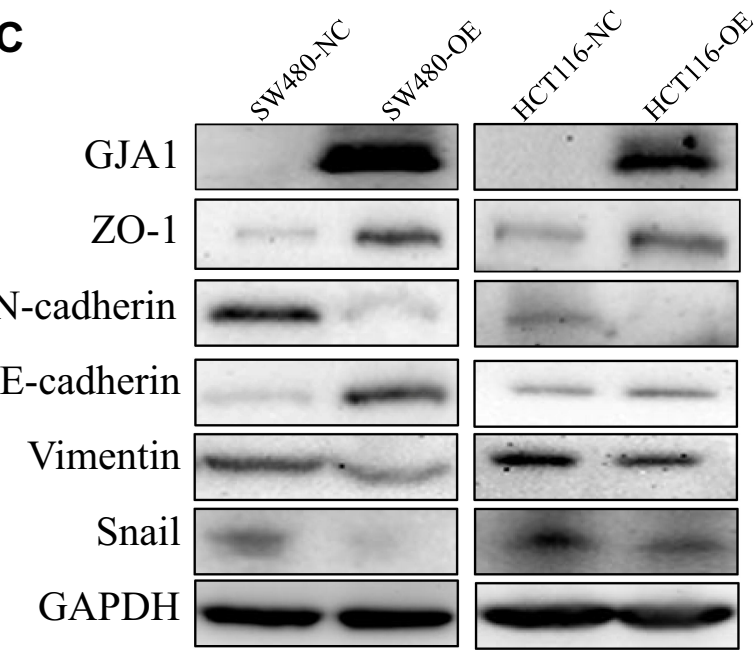

Figure 3 In vitro functional assay of GJAI in CRC cells. (A) Wound healing assay of control and GJAI-overexpressed CRC cells. Bar indicates $200 \mu \mathrm{m}$. (B) Transwell migration (left panel) and invasion assay (right panel) of control and GJAI-overexpressed CRC cells. (C) Expression levels of WNT pathway-associated proteins in control and GJAI-overexpressed were evaluated by Western blots. The statistical analysis is presented in Figure SI.

Moreover, we further measured the relationship of GJA1 with several chemokines and found that GJA1 was positively correlated with CCL5, CXCL5 and CXCL8 (Figure 5C), which were proved to be positively regulated by TAMs and TINs abundance. ${ }^{20,21}$ Most immune cells, including T cells, dendritic cells, and macrophages have been classified into different subpopulations that exert different, even opposite, roles in different disease contexts. ${ }^{22}$ TAM has two phenotypes, antitumorigenic M1 macrophages and protumorigenic M2 macrophages. Plenty of studies have demonstrated that M2-like macrophages polarization plays a vital role in promoting progression of CRC by activating several oncogenic pathways. ${ }^{23}$ Similarly, N1 and N2 neutrophils also display distinct functions in cancer. N1 neutrophils have potent anti-tumor activity 
A

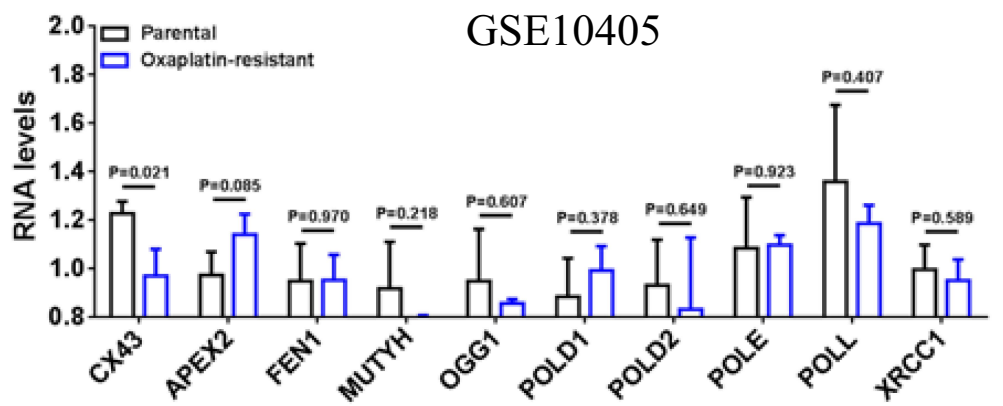

B

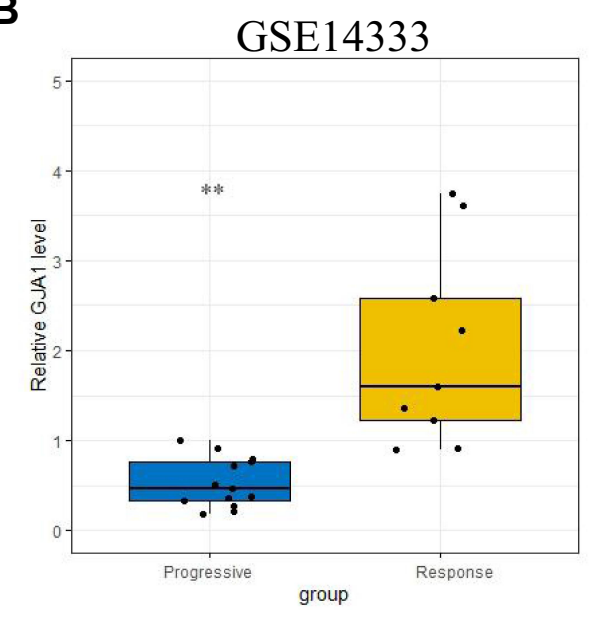

D

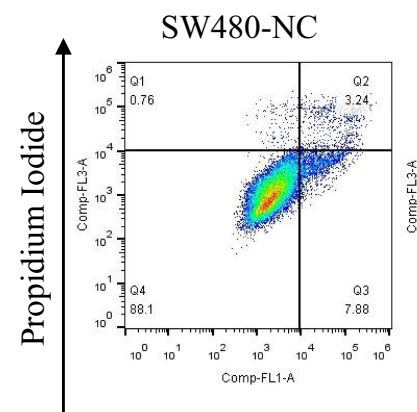

SW480-OE

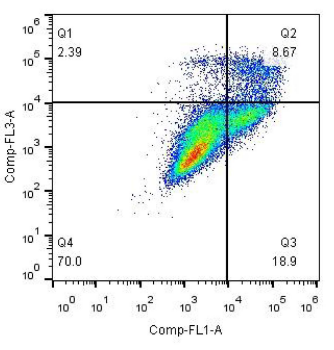

C

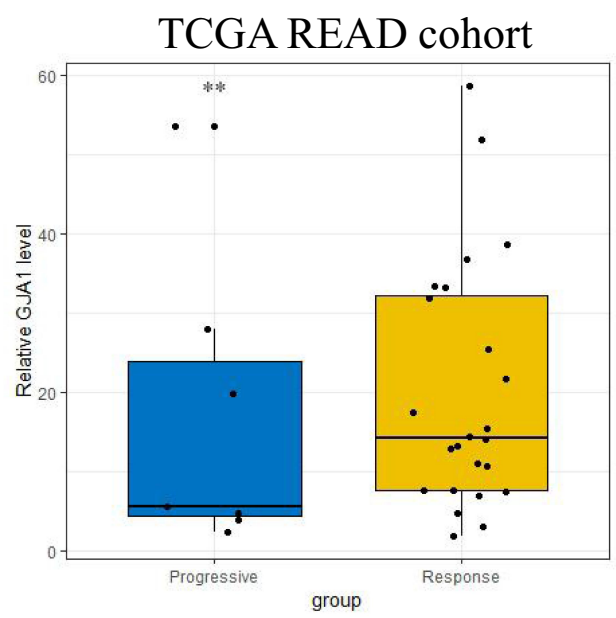

\section{Annexin-V}

Figure 4 GJAI promoted apoptosis of CRC cells. (A) Downregulation expression of GJAI protein (Cx43) was observed in oxaliplatin-resistant CRC cells. (B and C), RNAseq and clinical data from TCGA (B), and GEO (C) dataset showed that patients responding to neoadjuvant therapy exhibited higher expression of GJAI. (D) Flow cytometric assay showed GJAI-overexpressed cells showed better response to oxaliplatin. ** $P<0.0$ I by Students' $t$-test or Mann-Whitney U-test. Statistical analysis of flow cytometric assay was presented in Figure SI.

mainly due to their release of immunostimulatory cytokines such as CCL3, CXCL9 and CXCL10 which facilitaterecruitment and activation of CD8+ T cells. ${ }^{24}$ We presumed that GJA1 may increase the infiltration of M1 macrophages and $\mathrm{N} 1$ neutrophils. As expected, GJA1 positively correlated with cell markers of M1 macrophages and N1 neutrophils (Figure 5D and E), which confirm our hypothesis.

Moreover, we found that GJA1 also regulated infiltrating levels of $\mathrm{T}$ helper cells. As shown, GJA1 was positively associated with marker-genes of Th1 cells (Figure S2A) whereas it was negatively correlated with those of Th17 cells (Figure S2B), suggesting GJA1 not only recruited cytotoxic $\mathrm{T}$ cells but also enhanced their anti-tumor activity by gathering Th1 cells and expelling Th17 cells. Together, the above results further confirmed the hypothesis that GJA1 may regulate tumor immune response via interfering with multiple procedures. 
A

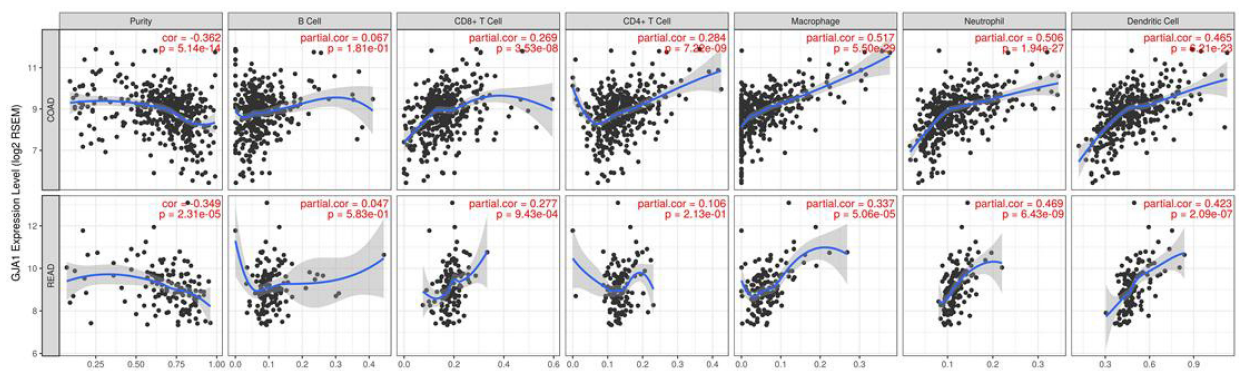

B
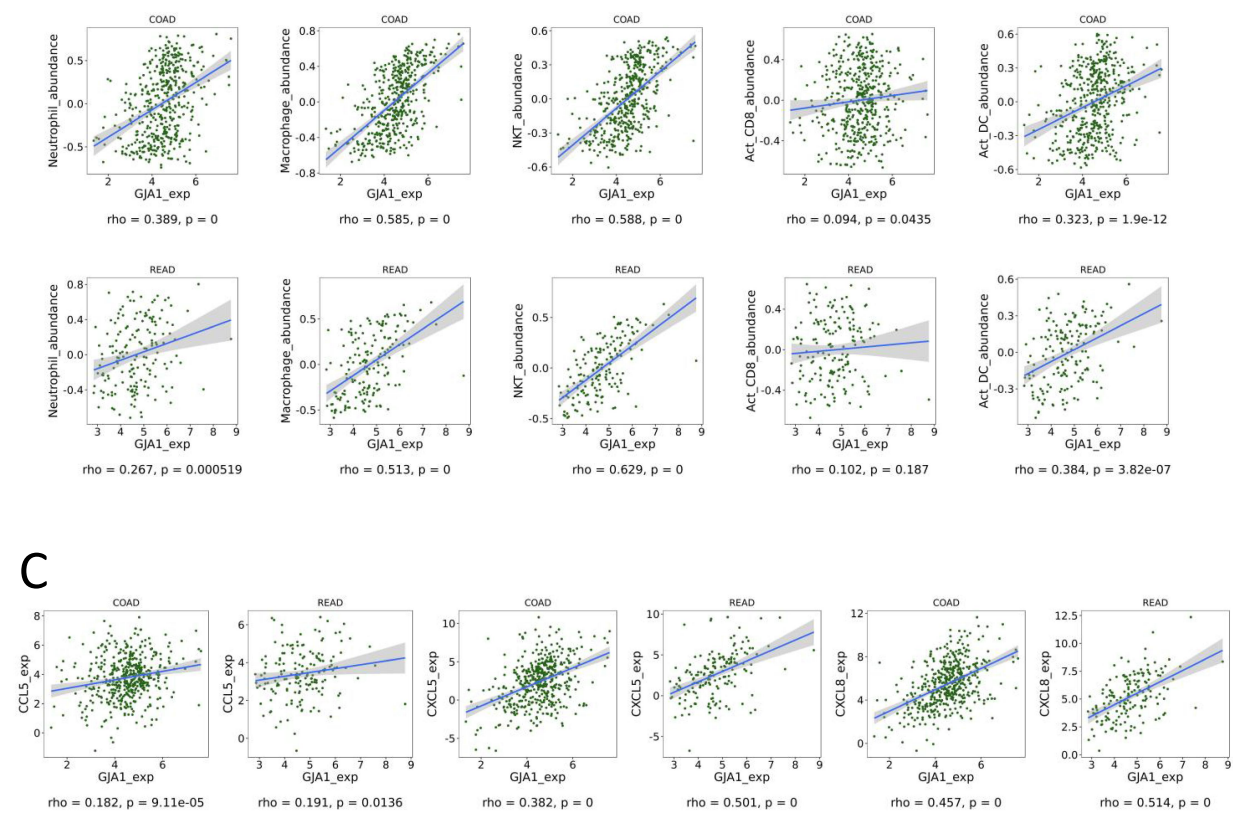

D

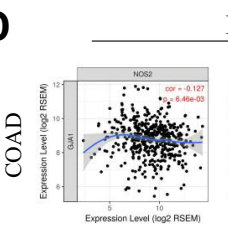

M1 macrophages
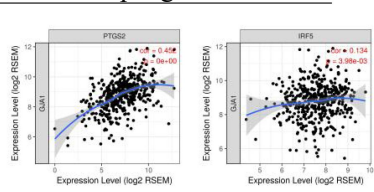

E

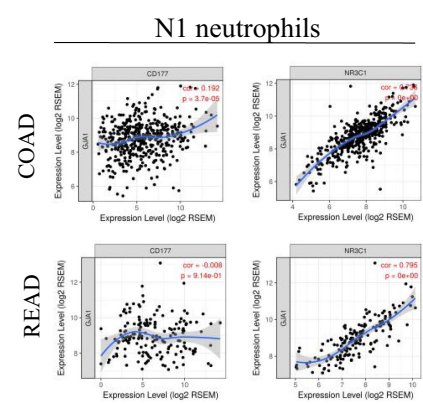

Figure 5 GJAI regulated tumor infiltrating immune cells. (A and B), correlation analysis of GJAI expression and tumor infiltrating immune cells performed by TIMER (A), and TISIDB (B) online tools. (C) Correlation analysis of GJAI expression and chemokines. (D and E), correlation analysis of GJAI expression and maker genes of MI macrophages (D), and NI neutrophils (E). R values and $P$ values were generated by Pearson correlation test.

\section{Copy Number Variation and Promoter Methylation Led to Downregulation of GJAI and Alteration of Tumor Infiltrating Immune Cells}

As the above results shown, loss of GJA1 promoted progression of $\mathrm{CRC}$ and resulted in unfavorable clinical outcomes. We then tried to investigate the mechanisms of downregulation of GJA1 in CRC. Several molecular events can lead to alterations in gene expression, such as copy number variation (CNV), deletion, and DNA methylation. As shown in Figure 6A, copy number variation and deletion of GJA1 coding gene happened in about 33\% CRC cases. Notably, although copy number variation 
showed negligible effects on GJA1 expression levels (Figure 6B-C), arm deletion resulted in decreased infiltration levels of $\mathrm{CD} 8+$ and $\mathrm{CD} 4+\mathrm{T}$ cells (Figure 6E), which suggested CNV represented a crucial mechanism by which GJA1 regulated tumor infiltrates. Moreover, DNA methylation is negatively correlated with GJA1 levels in CRC (Figure 6D). However, other mechanisms involved

A

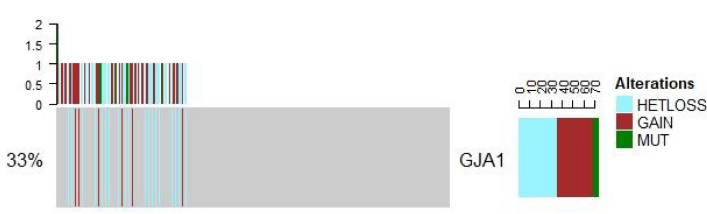

C

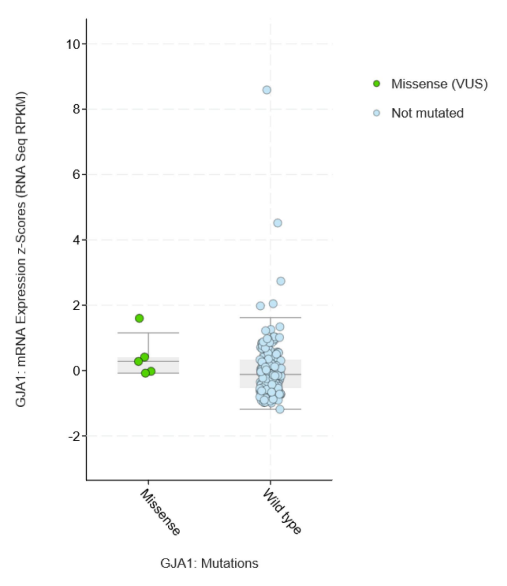

E

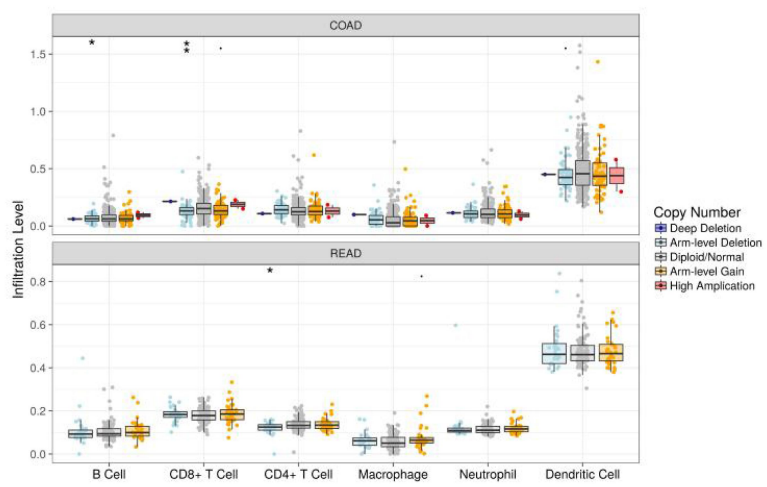

regulations of GJA1 protein, such as protein translation, ubiquitination, or subcellular location, need to be addressed in further studies.

\section{Discussion}

Colorectal cancer develops progressively through accumulation of genetic alterations such as activation of
B

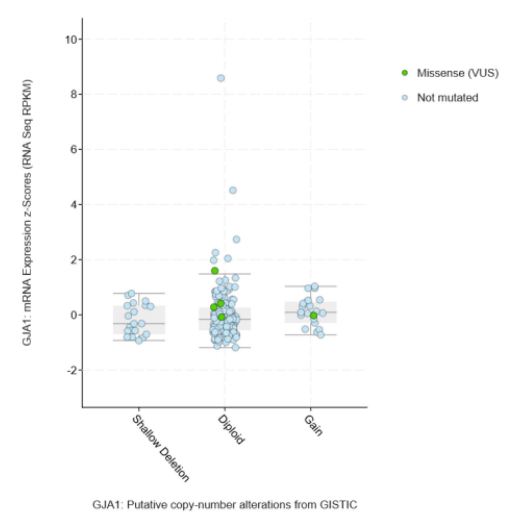

D

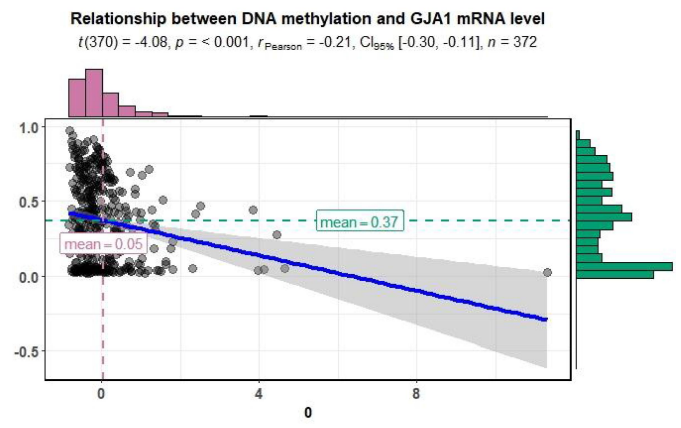

Figure 6 Genomic alterations of Gjal gene in CRC tissue. (A) Oncoprint showed CNV and mutation of Gjal gene in CRC tissue. (B and C) Effects of CNV (B), and mutation (C) on GJAI expression. (D) Correlation analysis of methylation of Gjal gene and GJAI mRNA level. (E) Effects of CNV of Gjal gene on infiltration levels of immune cells. Correlation analysis was performed with Pearson correlation analysis. Differences between CNV groups was calculated by one-way ANOVA. 
oncogenes and dysfunction of tumor suppressors. It has recently been reported that GJA1 is downregulated and related with relapse-free survival and overall survival in colorectal cancer. $^{25}$ Interestingly, there is also evidence of correlation of higher level of stromal expression of GJA1 with more advanced clinical stage of CRC. ${ }^{26,27}$ These contradictory findings indicate further investigation of GJA1 as a potential prognostic biomarker in CRC and the underlying mechanisms are warranted.

In the current study, we found the expression of GJA1 in colorectal tissues was significantly decreased compared with non-tumor tissues both in our study and the TCGA cohort. In terms of clinicopathological parameters, the expression of GJA1 was negatively related with the depth of invasion and distant metastasis, suggesting GJA1 might act as a tumor suppressor in CRC by inhibiting metastasis. Moreover, GJA1 expression is identified as a protective factor by multivariate Cox proportional hazards models, which was consistent with previous reports.

Then we investigated the function of GJA1 in CRC in vitro. Our data showed that forced expression of GJA1 significantly inhibited invasive ability of CRC cells, which accounted for the results of clinical-pathological correlation analysis. EMT is a biological process weakening of cell-to-cell adhesion, which enhances the cells migratory capacity and invasiveness. ${ }^{28}$ Previous studies demonstrated EMT was activated by several signaling pathways and promoted tumor initiation and progression. ${ }^{29}$ E-cadherin and N-cadherin are two important markers for EMT, which belong to the cadherin family. Loss of E-cad and overexpression of $\mathrm{N}$-cad are usually observed when EMT occurs. $^{28}$ We assumed that GJA1 might regulate CRC metastasis through modulating EMT because GJA1 was reported to be associated with $\beta$-catenin, a key component of the Wnt signaling pathway which could activate EMT. As expected, in our study, we found GJA1 overexpressed inhibiting cell wound healing, migration and invasion of CRC cell lines (Figure 3A and 3B). In addition, immunoblots showed that overexpression of GJA1 promoted expression of epithelial marker E-cadherin, ZO1 whereas decreased mesenchymal marker N-cadherin, Vimentin and Snail (Figure 3C). These results suggested GJA1 acted as a tumor suppressor gene in $\mathrm{CRC}$, and repressed cell migration and invasion through inhibiting EMT. However, how GJA1 downregulated these cell markers requires further investigation.

Another important aspect of this study was GJA1 correlated with diverse immune infiltrating cells in CRC.
Previous studies showed that infiltrated immune cells, including tumor-infiltrating neutrophils (TINs), lymphocytes, and CD8+T cells, were associated with the prognosis of CRC patients and response to checkpoint blockage therapy. ${ }^{10,12,30}$ Here, we reported that GJA1 was correlated with concomitant accumulation of several types of immune cells, including TINs, macrophages, CD8+ T cells and dendritic cells (Figure 5), at the peritumor microenvironment. Recently, there has been emerging interest in exploring the role of TINs in cancers. One study reported that TINs was positively related to prognosis and chemotherapy response in gastric cancer (GC), indicating a protective role of TINs in GC. ${ }^{13}$ However, in another clinical cohort TINs was a risk factor for CRC patients. $^{31}$ Interestingly, in experimental models, TINs can either promote or inhibit tumor progression via releasing cytokines and chemokines, just as tumor-associated macrophages (TAMs). ${ }^{20,21}$ The discrepancy of these studies reveals that there are different subgroups in TINs. It has been documented that neutrophils can polarize into $\mathrm{N} 1$ and N2 functional states: N1 neutrophils are antitumorigenic, whereas N2 neutrophils are protumorigenic. In our study, GJA1 is positively correlated with marker genes of N1 neutrophils, which indicated expression of GJA1 in CRC might cause accumulation of N1 neutrophils.

In summary, our results showed that GJA1 is a potential prognosis biomarker in patients with CRC. Besides, we further identified a positive relation between GJA1 and clinical outcomes in patients receiving chemotherapy. The results suggested that among patients receiving adjuvant chemotherapy, those with high GJA1 expression had longer OS compared with those with low GJA1, indicating that GJA1 could be an important factor for predicting the efficiency of chemotherapy. In addition, the ability of GJA1 to suppress CRC may be due to its association with TINs. The study is a retrospective research in nature and the number of patients receiving ACT is relatively small, and the full chemotherapy details were not available for the entire cohorts. Therefore, these results need a prospective, larger, multicentered randomized trial to validate.

\section{Abbreviations}

CRC, colorectal cancer; GJA1, gap junction alpha-1; qPCR, quantitative RT-PCR; NC, negative control; TAM, tumor-associated macrophages; TIN, tumor-infiltrating neutrophils. 


\section{Ethics Approval and Consent to Participate}

All participants provided written, informed consent and no participants had received any medication prior to sample collection under the guidelines of the Ethics Committee of Ruijin Hospital, which approved this study. This study was conducted in accordance with the Declaration of Helsinki.

\section{Acknowledgments}

The authors thank all members of the Shanghai Minimally Invasive Surgery Center for assistance in various aspects of this work.

\section{Funding}

This study has been supported by National Natural Science Foundation of China (No. 81802933).

\section{Disclosure}

The authors report no conflicts of interest in this work.

\section{References}

1. Siegel RL, Miller KD, Jemal A. Cancer statistics, 2019. CA Cancer J Clin. 2019;69(1):7-34. doi:10.3322/caac.21551

2. Allemani C, Matsuda T, Di Carlo V, et al. Global surveillance of trends in cancer survival 2000-14 (CONCORD-3): analysis of individual records for 37513025 patients diagnosed with one of 18 cancers from 322 population-based registries in 71 countries. The Lancet. 2018;391(10125):1023-1075. doi:10.1016/S0140-6736(17) 33326-3

3. Asmis TR, Saltz L. Systemic therapy for colon cancer. Gastroenterol Clin North Am. 2008;37(1):287-295. doi:10.1016/j. gtc.2007.12.005

4. Schedin P, Elias A. Multistep tumorigenesis and the microenvironment. Breast Cancer Research: BCR. 2004;6(2):93-101. doi:10.1186/ bcr772

5. Gava F, Rigal L, Mondesert O, et al. Gap junctions contribute to anchorage-independent clustering of breast cancer cells. Bmc Cancer. 2018;18(1):221. doi:10.1186/s12885-018-4148-5

6. Johnson RD, Camelliti P. Role of non-myocyte gap junctions and connexin hemichannels in cardiovascular health and disease: novel therapeutic targets? Int J Mol Sci. 2018;19(3):866. doi:10.3390/ ijms 19030866

7. Guy S, Geletu M, Arulanandam R, et al. Stat3 and gap junctions in normal and lung cancer cells. Cancers. 2014;6(2):646-662. doi:10.3390/cancers6020646

8. Falck E, Klingalevan K. Expression patterns of Phf5a/PHF5A and Gja1/GJA1 in rat and human endometrial cancer. Cancer Cell Int. 2013;13(1):43. doi:10.1186/1475-2867-13-43

9. Pagès F, Mlecnik B, Marliot F, et al. International validation of the consensus Immunoscore for the classification of colon cancer: a prognostic and accuracy study. Lancet. 2018;391(10135):10135. doi:10.1016/S0140-6736(18)30789-X

10. Restifo NP, Smyth MJ, Snyder A. Acquired resistance to immunotherapy and future challenges. Nat Rev Cancer. 2016;16(2):121. doi: $10.1038 / \mathrm{nrc} .2016 .2$

11. Coffelt SB, Wellenstein MD, Visser KED. Neutrophils in cancer: neutral no more. Nat Rev Cancer. 2016;16(7):431-446.
12. Naito Y, Saito K, Shiiba K, et al. CD8+ T cells infiltrated within cancer cell nests as a prognostic factor in human colorectal cancer. Cancer Res. 1998;58(16):3491-3494.

13. Zhang H, Liu H, Shen Z, et al. Tumor-infiltrating neutrophils is prognostic and predictive for postoperative adjuvant chemotherapy benefit in patients with gastric cancer. Ann Surg. 2016;267(2):1.

14. Wang TT, Zhao YL, Peng LS, et al. Tumour-activated neutrophils in gastric cancer foster immune suppression and disease progression through GM-CSF-PD-L1 pathway. Gut. 2017;66(11):1900-1911. doi:10.1136/gutjnl-2016-313075

15. Caruso RA, Fedele F, Rigoli L, et al. Mast cell interaction with tumor cells in small early gastric cancer: ultrastructural observations. Ultrastruct Pathol. 2009;21(2):9.

16. Hans-Gustaf L, Karl-Johan M. Prospects for the use of NK cells in immunotherapy of human cancer. Nat Rev Immunol. 2007;7(5):329339. doi:10.1038/nri2073

17. Mohamed SIA, Jantan I, Nafiah MA, et al. Dendritic cells pulsed with generated tumor cell lysate from Phyllanthus amarus Schum. \& Thonn. induces anti-tumor immune response. BMC Complement Altern Med. 2018;18(1):232. doi:10.1186/s12906-018-2296-4

18. Li T, Fan J, Wang B, et al. TIMER: a web server for comprehensive analysis of tumor-infiltrating immune cells. Cancer Res. 2017;77(21): e108-e10. doi:10.1158/0008-5472.CAN-17-0307

19. $\mathrm{Ru} \mathrm{B}$, Wong $\mathrm{CN}$, Tong Y, et al. TISIDB: an integrated repository portal for tumor-immune system interactions. Bioinformatics. 2019;35(20):4200-4202. doi:10.1093/bioinformatics/btz210

20. Shao-Lai Z, Zhi D, Zheng-Jun Z, et al. Overexpression of CXCL5 mediates neutrophil infiltration and indicates poor prognosis for hepatocellular carcinoma. Hepatology. 2012;56(6):2242-2254. doi: 10.1002/hep. 25907

21. Lechner MG, Liebertz DJ, Epstein AL. Characterization of cytokineinduced myeloid-derived suppressor cells from normal human peripheral blood mononuclear cells. J Immunol. 2010;185(4):22732284. doi:10.4049/jimmunol.1000901

22. Wang X, Qiu L, Li Z, et al. Understanding the multifaceted role of neutrophils in cancer and autoimmune diseases. Front Immunol. 2018;9:2456. doi:10.3389/fimmu.2018.02456

23. Engström A, Erlandsson A, Delbro D, et al. Conditioned media from M1 but not M2 macrophage phenotype inhibits proliferation of the colon cancer cell lines HT29 and CACO-2. J Biomed Sci. 2013;2:126.

24. Fridlender ZG, Albelda SM. Tumor-associated neutrophils: friend or foe? Carcinogenesis. 2012;33(5):949-955.

25. Wu J-I, Wang L-H. Emerging roles of gap junction proteins connexins in cancer metastasis, chemoresistance and clinical application. $J$ Biomed Sci. 2019;26(1):8. doi:10.1186/s12929-019-0497-x

26. Radić J, Krušlin B. Connexin 43 expression in primary colorectal carcinomas in patients with stage III and IV disease. Anticancer Res. 2016;36(5):2189.

27. Biscanin A, Ljubicic N, Boban M, et al. CX43 expression in colonic adenomas and surrounding mucosa is a marker of malignant potential. Anticancer Res. 2016;36(10):5437-5442. doi:10.21873/anticanres.11122

28. Kalluri R, Weinberg RA. The basics of epithelial-mesenchymal transition. J Clin Invest. 2009;119(6):1420-1428. doi:10.1172/jci39104

29. Kiesslich T, Pichler M, Neureiter D. Epigenetic control of epithelialmesenchymal-transition in human cancer. Mol clin oncol. 2013;1 (1):3-11. doi:10.3892/mco.2012.28

30. Nazemalhosseini-Mojarad E, Mohammadpour S, Torshizi Esafahani A. Intratumoral infiltrating lymphocytes correlate with improved survival in colorectal cancer patients: independent of oncogenetic features. J Cell Physiol. 2019;234(4):4768-4777. doi:10.1002/ jcp. 27273

31. Zhao JJ, Pan K, Wang W, et al. The prognostic value of tumorinfiltrating neutrophils in gastric adenocarcinoma after resection. PLoS One. 2012;7(3):e33655. doi:10.1371/journal.pone.0033655 


\section{Publish your work in this journal}

Cancer Management and Research is an international, peer-reviewed open access journal focusing on cancer research and the optimal use of preventative and integrated treatment interventions to achieve improved outcomes, enhanced survival and quality of life for the cancer patient.
The manuscript management system is completely online and includes a very quick and fair peer-review system, which is all easy to use. Visit http://www.dovepress.com/testimonials.php to read real quotes from published authors. 